\title{
TOPOLOGICAL CONJUGACY OF AFFINE TRANSFORMATIONS OF COMPACT ABELIAN GROUPS
}

BY

PETER WALTERS

0 . Introduction. We consider the following problem. If $X$ and $Y$ are compact connected metric abelian groups, $T=a+A$ an affine transformation of $X$ and $S=b+B$ an affine transformation of $Y$, what are necessary and sufficient conditions for every continuous mapping $g$ of $X$ onto $Y$ satisfying $g T=S g$ to be affine? Sufficient conditions are obtained in Theorem 3 in the case when the character group $\hat{Y}$ of $Y$ is polynomially annihilated by $B$ (see Definition 1). In Theorem 6 we show that these conditions are also necessary when $Y$ is a finite-dimensional group and in Theorem 8 we state (without proof) that this is also true in a more general setting. An example is given to show the assumption that $\hat{Y}$ be polynomially annihilated by $B$ cannot be dropped from Theorem 3. We also give an example which shows Theorem 6 to be false if $Y$ is not finite-dimensional but $\hat{Y}$ is polynomially annihilated by $B$.

Theorem 7 deals with the case when $T$ and $S$ are endomorphisms of an $n$ dimensional group. A conjugacy property of affine transformations with quasidiscrete spectrum is given in Theorem 4, and Theorem 5 gives information on continuous roots of affine transformations.

The results of this paper were proved in [10] for the cases when $X$ and $Y$ are finite-dimensional tori. The idea of using Theorem 1 was obtained from the paper [2].

1. Definitions and notations. Let $Y$ be a compact connected metric abelian (c.c.m.a.) group. We shall use additive notation in such groups. $\hat{Y}$ will denote the discrete torsion-free countable abelian character group of $Y$, and multiplicative notation will be used in $\hat{Y}$. $Y$ can be written as an inverse limit inv $\lim \left(Y_{m}, \sigma_{m}\right)$, where each $Y_{m}(m \geqq 1)$ is a finite-dimensional torus and $\sigma_{m}$ is a homomorphism of $Y_{m+1}$ onto $Y_{m}$. If $Y$ is $n$-dimensional then each $Y_{m}$ can be chosen to be an $n$ dimensional torus.

An affine transformation $S$ of a c.c.m.a. group $Y$ is a transformation of the form $S(y)=b+B(y), y \in Y$, where $b \in Y$ and $B$ is an endomorphism of $Y$ onto $Y$. We write $S=b+B$. Every affine transformation of $Y$ is continuous and preserves Haar measure. An endomorphism $B$ of $Y$ onto $Y$ induces a one-to-one dual endomorphism, which we also denote by $B$, of $\hat{Y}$ into $\hat{Y}$ defined by $(B \gamma)(y)=\gamma(B y), y \in Y$, $\gamma \in \hat{Y}$.

Received by the editors March 28, 1968. 
The affine transformation $S=b+B$ of $Y$ is ergodic if and only if $B^{n} \gamma=\gamma, \gamma \in \hat{Y}$, $n>0$, implies $B \gamma=\gamma$, and $[b,(B-I) Y]=Y$ where $[b,(B-I) Y]$ denotes the smallest closed subgroup of $Y$ containing $b$ and $(B-I) Y$. ( $I$ is the identity mapping of $Y$ [6].) Also, $S=b+B$ is ergodic if and only if there exists $y_{0} \in Y$ such that $\left\{S^{n}\left(y_{0}\right) \mid n \geqq 0\right\}$ is dense in $Y$ [10]. From the first condition it follows that an endomorphism $B$ of $Y$ onto $Y$ is ergodic if and only if $B^{n} \gamma=\gamma, \gamma \in \hat{Y}, n>0$, implies $\gamma=1$ (see also [5]). Also from the first condition we have that $S=b+B$ is strong mixing if and only if $B$ is ergodic (see also [3]).

$\boldsymbol{R}^{n}$ will denote real Euclidean $n$-space, $\boldsymbol{Z}^{n}$ the subgroup of $\boldsymbol{R}^{n}$ of points with integer coordinates and $\boldsymbol{K}^{n}=\boldsymbol{R}^{n} / \boldsymbol{Z}^{n}$ the $n$-dimensional torus. $\mathscr{R}(\mu)$ and $\mathscr{I}(\mu)$ will denote the real and imaginary parts of the complex number $\mu$, and if $P(x)=$ $\left(P_{1}(x), P_{2}(x), \ldots, P_{n}(x)\right)$ is a transformation from a set $X$ to complex $n$-space $C^{n}$, then $\mathscr{R} P$ and $\mathscr{I} P$ will denote the transformations of $X$ to $R^{n}$ defined by $(\mathscr{R} P)(x)$ $=\left(\mathscr{R} P_{1}(x), \ldots, \mathscr{R} P_{n}(x)\right)$ and $(\mathscr{I} P)(x)=\left(\mathscr{I} P_{1}(x), \ldots, \mathscr{I} P_{n}(x)\right)$ respectively.

$\boldsymbol{Q}$ will denote the field of rational numbers and $Q[\theta]$ the algebra of all polynomials in $\theta$ with coefficients from $Q$.

\section{Preliminary results.}

THEOREM 1 (VAN KAMPEN). Let $Y$ be a c.c.m.a. group and $f$ be a continuous function from $Y$ to the set of complex numbers of unit modulus. Then $f$ can be expressed in the form $f(y)=\alpha(y) e^{i \phi(y)}, y \in Y$, where $\alpha \in \hat{Y}$ is uniquely determined by $f, \phi: Y \rightarrow R$ is continuous and is uniquely determined up to an additive constant.

Proofs of this theorem can be found in [9] and [2]. The following is immediate from Theorem 1.

COROllaRY 1.1. Let $X$ and $Y$ be c.c.m.a. groups and $g: X \rightarrow Y$ a continuous mapping. For each $\gamma \in \hat{Y}$ there exists a unique $\alpha_{\gamma} \in \hat{X}$ and a continuous mapping $\phi_{\gamma}: X \rightarrow R$ unique up to an additive constant, such that $(\gamma \circ g)(x)=\alpha_{\gamma}(x) \exp \left[i \phi_{\gamma}(x)\right]$, $x \in X$. Furthermore $\exp \left[i \phi_{\gamma \gamma^{1}}(x)\right]=\exp \left[i \phi_{\gamma}(x)\right] \cdot \exp \left[i \phi_{\gamma^{1}}(x)\right], x \in X, \gamma, \gamma^{1} \in \hat{Y}$.

THEOREM 2. Let $X$ and $Y$ be c.c.m.a. groups and suppose that for every $\gamma \in \hat{Y}$ there exists a continuous mapping $\phi_{\gamma}: X \rightarrow R$ such that $\phi_{\gamma \gamma^{1}}=\phi_{\gamma}+\phi_{\gamma^{1}}, \gamma, \gamma^{1} \in \hat{Y}$. Then there exists a continuous mapping $u: X \rightarrow Y$ such that $\gamma \circ u(x)=\exp \left[i \phi_{\gamma}(x)\right]$, $x \in X, \gamma \in \hat{Y}$, and $u$ is homotopic to a constant.

Proof. For each $x \in X$ the mapping $\gamma \rightarrow \exp \left[i \phi_{\gamma}(x)\right]$ is a character of $\hat{Y}$ and therefore there exists $y_{x} \in Y$ such that $\gamma\left(y_{x}\right)=\exp \left[i \phi_{y}(x)\right]$. Define $u: X \rightarrow Y$ by $u(x)=y_{x} \cdot u$ is clearly continuous.

For each $x \in X$ and each $t \in[0,1]$ the mapping $\gamma \rightarrow \exp \left[i t \phi_{\gamma}(x)\right]$ is a character of $\hat{Y}$ and, as above in the case $t=1$, there exists a continuous mapping $u_{t}: X \rightarrow Y$ such that $\gamma\left(u_{t}(x)\right)=\exp \left[i t \phi_{\gamma}(x)\right], x \in X, \gamma \in \hat{Y} . u_{t}$ is a homotopy between $u$ and a constant. 
3. Topological conjugacy and groups with polynomially annihilated character groups. Let $Y$ be a c.c.m.a. group and $B$ an endomorphism of $Y$ onto $Y$. Let $p(\theta)=n_{0}+n_{1} \theta+\cdots+n_{k} \theta^{k}$ be a polynomial over $Z$. We shall say that $p$ is an annihilating polynomial of $\gamma \in \hat{Y}$ with respect to $B$ if $\gamma^{n_{0}} \cdot B \gamma^{n_{1}} \cdots B^{k} \gamma^{n_{k}}=1$.

Suppose $\gamma \in \hat{Y}$ has a nontrivial annihilating polynomial with respect to $B$. Let $M_{\gamma}$ denote the set of all polynomials over $\boldsymbol{Q}$ some integral multiple of which is an annihilating polynomial of $\gamma$ with respect to $B . M_{\gamma}$ is an ideal in $Q[\theta]$ and therefore there exists a unique monic polynomial $q_{\gamma} \in Q[\theta]$ such that $M_{\gamma}$ is the principal ideal generated by $q_{\gamma}[8, \mathrm{p} .121]$. If $q_{\gamma}(\theta)=s_{0}+s_{1} \theta+\cdots+s_{l-1} \theta^{l-1}+\theta^{l}$ then $s_{0} \neq 0$ for otherwise $q_{\gamma}^{1}(\theta)=s_{1}+s_{2} \theta+\cdots+s_{l-1} \theta^{l-2}+\theta^{l-1}$ would be a monic polynomial generating $M_{\gamma}$. If $n_{q}$ is the lowest common denominator of the nonzero members of $s_{0}, s_{1}, \ldots, s_{l-1}$ then $p_{\gamma}(\theta)=n_{q} s_{0}+n_{q} s_{1} \theta+\cdots+n_{q} \theta^{l}$ is a polynomial over $Z$ which will be called the minimal annihilating polynomial of $\gamma$ with respect to $B$.

Definition 1. Let $Y$ be a c.c.m.a. group and $B$ an endomorphism of $Y$ onto $Y$. We say that $\hat{Y}$ is polynomially annihilated by $B$ if every element of $\hat{Y}$ has a nontrivial annihilating polynomial with respect to $B$.

If $Y$ is an $n$-dimensional c.c.m.a. group then $\hat{Y}$ is polynomially annihilated by any endomorphism $B$ of $Y$ onto $Y$. This follows because $\dot{Y}$ is isomorphic to a subgroup of the additive group $Q^{n}$ (the direct sum of $n$ copies of $Q$ ) and therefore the one-toone endomorphism $B$ of $\hat{Y}$ corresponds to an $n \times n$ matrix with rational entries and nonzero determinant. The Cayley-Hamilton theorem shows that some integral multiple of the characteristic polynomial of this matrix is an annihilating polynomial, with respect to $B$, of every element of $\hat{Y}$. If $q(\theta)=s_{0}+s_{1} \theta+\cdots+\theta^{n}$ is the characteristic polynomial of some matrix representation of $B$ and if $n_{q}$ is the lowest common denominator of the nonzero members of $s_{0}, s_{1}, \ldots, s_{n-1}$, then the polynomial $p(\theta)=n_{q} s_{0}+n_{q} s_{1} \theta+\cdots+n_{q} \theta^{n}$ is a polynomial over $Z$, which will be called the annihilating polynomial of $\hat{Y}$ with respect to $B$. This polynomial is independent of the matrix representation of $B$.

The following lemma will be used in the proof of Theorem 3 .

Lemma 1. Let $X$ be a c.c.m.a. group and $T=a+A$ an affine transformation of $X$. Suppose $\Phi: X \rightarrow R^{n}$ is a nonconstant continuous function and $M$ is a linear transformation of $\boldsymbol{R}^{n}$ such that $\Phi(T x)=M \Phi(x)+d, x \in X$, where $d \in \boldsymbol{R}^{n}$. Then there exists $\delta \in \hat{X}, \delta \neq 1$, and a root $\lambda$, with $|\lambda|=1$, of the characteristic equation of $M$ such that $A^{p} \delta=\delta$ for some $p \geqq 1$ and $\delta\left(a+A(a)+\cdots+A^{p-1}(a)\right)=\lambda^{p}$ for all such $p$.

Proof. We consider $\boldsymbol{R}^{n}$ as a subset of $\boldsymbol{C}^{n}$ (complex $n$-space) in the usual way and complexify $M$. There exists an invertible linear transformation $U$ of $\boldsymbol{C}^{n}$ such that $U^{-1} M U=D_{M}$, the Jordan normal form of the linear transformation $M$. Therefore $U^{-1} \Phi(T x)=D_{M} U^{-1} \Phi(x)+U^{-1} d, x \in X$ If $w_{1}, w_{2}, \ldots, w_{n}$ denotes the fixed basis of $\boldsymbol{R}^{n}$ then $w_{1}, w_{2}, \ldots, w_{n}$ is also a basis, using complex coefficients for $\boldsymbol{C}^{n}$. Suppose $U^{-1} \Phi(x)=\sum_{i=1}^{n} f_{i}(x) w_{i}$. Each $f_{i}: X \rightarrow C$ is continuous, and if $i_{0}$ is the least positive integer for which $f_{i_{0}}$ is nonconstant then $f_{i_{0}}(T x)=\lambda f_{i_{0}}(x)+e, x \in X$, where $e \in C$ 
and $\lambda$ is an eigenvalue of $M$. If $l: X \rightarrow C$ is defined by $l(x)=f_{i_{0}}(x)-\int_{X} f_{i_{0}}(x) d m$, where $m$ denotes Haar measure on $X$, then $l(T x)=\lambda l(x)$ and $l$ is nonconstant and continuous. Since $T$ maps $X$ onto $X \sup _{X}|l(T X)|=|\lambda| \sup _{X}|l(x)|$ implies $|\lambda|=1$. But $l \in L^{2}(X)$ and therefore $l(x)=\sum_{i} b_{i} \delta_{i}(x)$ ( $L^{2}$ convergence) where $\delta_{i} \in \hat{X}$ and $\sum_{i}\left|b_{i}\right|^{2}<\infty$. From the equation $l\left(T^{p} x\right)=\lambda^{p} l(x), p \geqq 1$, we have

$$
\sum_{i} b_{i} \delta_{i}\left(a+A a+\cdots+A^{p-1} a\right) \delta_{i}\left(A^{p} x\right)=\lambda^{p} \sum_{i} b_{i} \delta_{i}(x) \quad\left(L^{2} \text { convergence }\right) .
$$

If $\delta_{i}, A \delta_{i}, A^{2} \delta_{i}, \ldots$ are all distinct then $b_{i}=0$ for otherwise the condition $\sum_{i}\left|b_{i}\right|^{2}<\infty$ is violated. Therefore $b_{i} \neq 0$ implies $A^{p} \delta_{i}=\delta_{i}$ for some $p \geqq 1$ and when this occurs $\delta_{i}\left(a+A a+\cdots+A^{p-1} a\right)=\lambda^{p}$. Since $l(x)$ is nonconstant there must be some $\delta_{i} \in \hat{X}$, $\delta_{i} \neq 1$, with this property.

TheOREM 3. Let $X$ and $Y$ be c.c.m.a. groups. Let $T=a+A$ be an affine transformation of $X$ and $S=b+B$ an affine transformation of $Y$. Suppose further that $\hat{Y}$ is polynomially annihilated by $B$. If there exists a nonaffine continuous mapping $g: X \rightarrow Y$ such that $g T=S g$ then there exists $\delta \in \hat{X}, \delta \neq 1$, and a root $\lambda$, with $|\lambda|=1$, of the minimal annihilating polynomial with respect to $B$ of some element of $\hat{Y}$, such that $A^{p} \delta=\delta$ for some $p \geqq 1$ and $\delta\left(a+A a+\cdots+A^{p-1} a\right)=\lambda^{p}$ for all such $p$.

Proof. Using the notation of Corollary 1.1, for $\gamma \in \hat{Y}$ let

$$
(\gamma \circ g)(x)=\alpha_{\gamma}(x) \exp \left[i \phi_{\gamma}(x)\right]
$$

where $\alpha_{\gamma} \in \hat{X}$ and $\phi_{\gamma}: X \rightarrow R$ is continuous. Since $g$ is nonaffine there exists $\gamma_{0} \in \hat{Y}$ such that $\phi_{\gamma_{0}}$ is nonconstant. Applying $\gamma \in \hat{Y}$ to the equation $g T=S g$ and using the uniqueness asserted in Corollary 1.1 we have $\alpha_{\gamma}(a) \exp \left[i \phi_{\gamma}(T x)\right]=\gamma(b) \exp \left[i \phi_{B \gamma}(x)\right]$. Since $X$ is connected this implies

$$
\phi_{\gamma}(T x)=\phi_{B \gamma}(x)+c_{\gamma}, \quad x \in X,
$$

where $c_{\gamma} \in \boldsymbol{R}$. Suppose that $p_{\gamma_{0}}$, the minimal annihilating polynomial of $\gamma_{0}$ with respect to $B$, is of degree $n$. Define $\Phi: X \rightarrow \boldsymbol{R}^{n}$ by

$$
\Phi(x)=\left[\begin{array}{c}
\phi_{\gamma_{0}}(x) \\
\phi_{B \gamma_{0}}(x) \\
\vdots \\
\phi_{B^{n-1} \gamma_{0}}(x)
\end{array}\right], \quad x \in X .
$$

$\Phi$ is nonconstant and continuous. If $p_{\gamma_{0}}(\theta)=m_{0}+m_{1} \theta+\cdots+m_{n} \theta^{n}, m_{i} \in \boldsymbol{Z}$ $(1 \leqq i \leqq n), m_{n} \neq 0$, then using the connectedness of $X$ we have that $m_{n} \phi_{B^{n} \gamma_{0}}(x)$ $+m_{n-1} \phi_{B-1} \gamma_{0}+\cdots+m_{0} \phi_{\gamma_{0}}(x)$ is a constant mapping. Let $M$ denote the linear transformation of $\boldsymbol{R}^{n}$ given by the matrix 


$$
\left[\begin{array}{ccccc}
0 & 1 & \cdots & 0 & 0 \\
0 & 0 & \cdots & 0 & 0 \\
\vdots & \vdots & & \vdots & \vdots \\
0 & 0 & & 1 & 0 \\
0 & 0 & \cdots & 0 & 1 \\
-m_{0} / m_{n} & -m_{1} / m_{n} & \cdots & -m_{n-2} / m_{n} & -m_{n-1} / m_{n}
\end{array}\right] .
$$

Then $\Phi(T x)=M \Phi(x)+d, x \in X$, where $d \in \boldsymbol{R}^{n}$, and the result follows from Lemma 1 since $p_{\gamma_{0}}$ is the characteristic polynomial of $M$.

Corollary 3.1. Let $X, Y, T, S$ be as in Theorem 3 with the additional assumption that $T$ is ergodic. If there is a nonaffine continuous mapping $g: X \rightarrow Y$ such that $g T=S g$ then there exists $\delta \in \hat{X}, \delta \neq 1$, and a root $\lambda$, with $|\lambda|=1$, of the minimal annihilating polynomial with respect to $B$ of some element of $\hat{Y}$, such that $\lambda$ is not a root of unity, $A \delta=\delta$ and $\delta(a)=\lambda$.

Hence if $T$ is strong mixing, all continuous mappings $g: X \rightarrow Y$ such that $g T=S g$ are affine.

Proof. Let $\delta$ be the element of $\hat{X}$ and $\lambda$ the complex number which are determined by Theorem 3. Since $A^{p} \delta=\delta$ for some $p \geqq 1$, the ergodicity of $T$ implies $A \delta=\delta$ and hence $\delta(a)=\lambda$. If $\lambda$ were a root of unity then since $[a,(A-I) X]=X, \delta$ would only assume a finite number of values on $X$ and would have to be the identity character.

Lastly, if $T$ is strong mixing then $A$ is ergodic and there is no $\delta \in \hat{X}, \delta \neq 1$, with $A \delta=\delta$.

Theorem 2 of Arov [2] follows from Corollary 3.1.

The notion of a measure-preserving transformation with quasi-discrete spectrum has been defined by Abramov [1], and the notion of a homeomorphism with quasi-discrete spectrum has been defined by Hahn and Parry [4]. An ergodic affine transformation $S=b+B$ of a c.c.m.a. group $Y$ has quasi-discrete spectrum as a (Haar) measure-preserving transformation if and only if it has quasi-discrete spectrum as a homeomorphism. In fact $S=b+B$, assumed to be ergodic, has quasi-discrete spectrum in either sense if and only if $\bigcap_{n=0}^{\infty}(B-I)^{n} Y=\{0\}$, where $I$ denotes the identity mapping of $Y$ [7]. The following result extends Theorem 6 of the paper [4].

THeOREM 4. Let $X$ and $Y$ be c.c.m.a. groups and let $T=a+A$ be an ergodic affine transformation of $X$ and $S=b+B$ an ergodic affine transformation of $Y$. If $S$ has quasi-discrete spectrum then all continuous mappings $g: X \rightarrow Y$ satisfying $g T=S g$ are affine.

Proof. Let $\gamma \in \hat{Y}$. There exists $n \geqq 1$ such that $(\theta-1)^{n}$ is an annihilating polynomial of $\gamma$ with respect to $B$. It follows that the roots of the minimal annihilating 
polynomial of $\gamma$ with respect to $B$ are equal to 1 . The result follows from Corollary 3.1.

THEOREM 5. Let $Y$ be a c.c.m.a. group and $S=b+B$ a strong mixing affine transformation of $Y$ such that $\hat{Y}$ is polynomially annihilated by $B$. Then every continuous pth root $(p \geqq 1)$ of $S$ is an affine transformation and $S$ has a continuous pth root if and only if there is an endomorphism $C$ of $Y$ onto $Y$ with $C^{p}=B$.

Proof. Suppose $g$ is a continuous $p$ th root of $S$. Then $g S=S g$ and $g$ is affine by Corollary 3.1. Since $S$ is strong mixing $B$ is ergodic and therefore $(B-I) Y=Y$. Choose $y_{0} \in Y$ so that $(B-I) y_{0}=b$ and the homeomorphism $h: Y \rightarrow Y$, defined by $h(y)=y_{0}+y$, satisfies $h S=B h$. Therefore $S$ has a continuous $p$ th root if and only if $B$ has a continuous $p$ th root. Any continuous $p$ th root of $B$ is affine and the $p$ th power of its endomorphism part will be $B$. Conversely if $C$ is an endomorphism of $Y$ onto $Y$ with $C^{p}=B$ then $C$ is a continuous $p$ th root of $B$.

As a special case of Corollary 3.1 we have the following result. If $Y$ is a c.c.m.a. group and $B$ is an ergodic endomorphism of $Y$ onto $Y$ which polynomially annihilates $\hat{Y}$, then every continuous mapping commuting with $B$ is affine. The example below shows that this result is false (and therefore Theorem 3 is false) if the assumption that $\hat{Y}$ be polynomially annihilated by $B$ is dropped.

Let $K^{\infty}$ denote the two-sided infinite-dimensional torus (i.e. the two-sided infinite direct sum of copies of $\boldsymbol{K}$ ) and let $B$ denote the shift automorphism of $\boldsymbol{K}^{\infty}$ defined by $(B z)_{n}=z_{n+1}$ if $z=\left(z_{n}\right)$. No nontrivial element of $\hat{\boldsymbol{K}}^{\infty}$ is polynomially annihilated by $B$. Let $f: \boldsymbol{K} \rightarrow \boldsymbol{K}$ be any homeomorphism and define $F: \boldsymbol{K}^{\infty} \rightarrow \boldsymbol{K}^{\infty}$ by $(F(z))_{n}=f\left(z_{n}\right),-\infty<n<\infty . F$ is a homeomorphism and $F B=B F$. Moreover $F$ can be chosen to be nonaffine by choosing $f$ nonaffine.

It would be interesting to know if the condition that $\hat{Y}$ be polynomially annihilated by $B$ follows from the fact that every continuous mapping commuting with $B$ ( $B$ ergodic) is affine.

\section{Converses of Theorem 3.}

LemMA 2. Let $X$ and $Y$ be c.c.m.a. groups and let them be represented as $X=\operatorname{inv} \lim \left(X_{q}, \tau_{q}\right)$ and $Y=\operatorname{inv} \lim \left(Y_{m}, \sigma_{m}\right)$ where $X_{q}(q \geqq 1)$ and $Y_{m}(m \geqq 1)$ are finite-dimensional tori. Let $C$ be a homomorphism of $X$ onto $Y$ and let $u: X \rightarrow Y$ be a continuous mapping which depends only on $X_{k_{0}}$ and which is homotopic to a constant by a homotopy which depends only on $X_{k_{0}}$. Then $C+u$ maps $X$ onio $Y$.

Proof. Let $C_{m}$ and $u_{m}(m \geqq 1)$ denote the mappings of $X$ to $Y_{m}$ obtained by projecting $C$ and $u$ onto $Y_{m}$. $C+u$ will map $X$ onto $Y$ if and only if $C_{m}+u_{m}$ maps $X$ onto $Y_{m}$ for each $m \geqq 1$. For each $m \geqq 1$ there exists $q_{m} \geqq 1$ such that $C_{m}$ only depends on $X_{q_{n}}$. Let $k_{m}=\max \left(q_{m}, k_{0}\right)$. Then $C_{m}$ can be considered as a homomorphism of $X_{k_{m}}$ onto $Y_{m}$ and $u_{m}$ can be considered as a continuous mapping of $X_{k_{m}}$ into $Y_{m}$ which is homotopic (on $X_{k_{m}}$ ) to a constant. The result will follow if we can show that whenever $C$ is a homomorphism of $\boldsymbol{K}^{n}$ onto $\boldsymbol{K}^{m}$ and $u: \boldsymbol{K}^{n} \rightarrow \boldsymbol{K}^{m}$ is a continuous 
mapping homotopic to a constant then $C+u$ maps $\boldsymbol{K}^{n}$ onto $\boldsymbol{K}^{m}$. However this result follows from Lemma 1 of [10].

Lemma 3. Let $P: \boldsymbol{R}^{n} \rightarrow \boldsymbol{R}^{n}$ be a continuous mapping such that $P(v+\tau)=P(v)$, $v \in \boldsymbol{R}^{n}, \tau \in \boldsymbol{Z}^{n}$ and $\left\|P(v)-P\left(v^{\prime}\right)\right\|<\left\|v-v^{\prime}\right\|, v, v^{\prime} \in \boldsymbol{R}^{n}$ where $\|\cdot\|$ denotes the usual norm in $\boldsymbol{R}^{n}$. Let $\psi: \boldsymbol{K}^{n} \rightarrow \boldsymbol{K}^{n}$ be the continuous mapping defined by $\psi \pi=\pi P$, where $\pi: \boldsymbol{R}^{n} \rightarrow \boldsymbol{K}^{n}$ is the natural projection. Then $I+\psi$ is a one-to-one mapping of $\boldsymbol{K}^{n}$. (I denotes the identity mapping of $\boldsymbol{K}^{n}$.)

Proof. Let $I^{\prime}$ denote the identity mapping of $\boldsymbol{R}^{n}$. $I^{\prime}+P$ is a one-to-one mapping because $v+P(v)=v^{\prime}+P\left(v^{\prime}\right)$ implies $v-v^{\prime}=P\left(v^{\prime}\right)-P(v)$ and hence $v=v^{\prime}$. Suppose $(I+\psi) \pi(v)=(I+\psi) \pi\left(v^{\prime}\right)$. Then $\pi\left(I^{\prime}+P\right)(v)=\pi\left(I^{\prime}+P\right)\left(v^{\prime}\right)$ and

$$
\begin{aligned}
\left(I^{\prime}+P\right)(v) & =\left(I^{\prime}+P\right)\left(v^{\prime}\right)+\tau, \quad \tau \in Z^{n} \\
& =\left(I^{\prime}+P\right)\left(v^{\prime}+\tau\right) .
\end{aligned}
$$

Therefore $v=v^{\prime}+\tau$ and $\pi(v)=\pi\left(v^{\prime}\right)$. This proves that $I+\psi$ is one-to-one.

The following theorem gives a converse to Theorem 3 in the cases when $Y$ is a finite-dimensional group.

THEOREM 6. Let $X$ and $Y$ be c.c.m.a. groups and suppose that $Y$ is $n$-dimensional. Let $T=a+A$ be an affine transformation of $X, S=b+B$ an affine transformation of $Y$ and suppose there exists a continuous mapping $h$ of $X$ onto $Y$ such that $h T=S h$. Suppose further there exists $\delta \in \hat{X}, \delta \neq 1$, and a root $\lambda$, with $|\lambda|=1$, of the annihilating polynomial of $\hat{Y}$ with respect to $B$ such that $A^{p} \delta=\delta$ for some $p \geqq 1$ and $\delta(a+A(a)+$ $\left.\cdots+A^{p-1}(a)\right)=\lambda^{p}$ for all such $p$. Then there exists a nonaffine continuous mapping $g$ of $X$ onto $Y$ such that $g T=S g$. Moreover, if $h$ is given to be a homeomorphism then $g$ can be chosen to be a homeomorphism.

Proof. We may as well assume that the given mapping $h$ is affine or there is nothing to prove. Suppose $h=c+C$, where $c \in Y$ and $C$ is a homomorphism of $X$ onto $Y$. We shall use Theorem 2 and to do this we have to construct continuous mappings $\phi_{\gamma}: X \rightarrow R$ for each $\gamma \in \hat{Y}$.

Since $Y$ is $n$-dimensional $\hat{Y}$ is isomorphic to a subgroup $Q_{Y}^{n}$ of the additive group $Q^{n}$ and we can choose $Q_{Y}^{n}$ so that if $d_{i}=\left(d_{i 1}, d_{i 2}, \ldots, d_{i n}\right)$ where

$$
\begin{aligned}
d_{i j}=1 & \text { if } i=j, \\
=0 & \text { if } i \neq j,
\end{aligned}
$$

then $d_{i} \in Q_{Y}^{n}(1 \leqq i \leqq n)$. Let $[M]$ be the matrix (with rational entries) representing the action of $B$ on $Q_{Y}^{n}$, and let $\gamma_{i} \in \hat{Y}$ correspond under the above isomorphism to $d_{i} \in Q_{Y}^{n}(1 \leqq i \leqq n)$. Let $M$ denote the linear transformation of $\boldsymbol{R}^{n}$ induced by the matrix $[M]$.

Suppose that $p$ is the smallest positive integer such that $A^{p} \delta=\delta$. Define $f: X \rightarrow C$ by

$$
f(x)=\sum_{j=0}^{p-1} \frac{\delta\left(a+A(a)+\cdots+A^{j-1}(a)\right)}{\lambda^{j}} \delta\left(A^{j} x\right), \quad x \in X,
$$


$f$ is a nonconstant continuous function satisfying $f(T x)=\lambda f(x), x \in X$. If $w_{1}, w_{2}, \ldots, w_{n}$ denotes the fixed basis of $\boldsymbol{R}^{n}$ it is also a basis for $\boldsymbol{C}^{n}$. Let $U$ be the invertible linear transformation of $C^{n}$ such that $U^{-1} M U=D_{M}$, the Jordan normal form of the complexified linear transformation $M$. Let $j_{0}$ be the largest integer for which $w_{j_{0}}$ corresponds to the eigenvalue $\lambda$ of $D_{M}$. Then $U\left(f(x) w_{j_{0}}\right)$ is nonconstant and so either $\mathscr{R} U\left(f(x) w_{j_{0}}\right)$ or $\mathscr{I} U\left(f(x) w_{j_{0}}\right)$ is nonconstant. Suppose, without loss of generality, that $\mathscr{R} U\left(f(x) w_{j_{0}}\right)$ is nonconstant and define the mappings $\phi_{\gamma_{i}}: X \rightarrow \boldsymbol{R}$ by

$$
\sum_{i=1}^{n} \phi_{\gamma_{i}}(x) w_{i}=\mathscr{R} U\left(f(x) w_{j_{0}}\right), \quad x \in X
$$

Let $\gamma \in \hat{Y}$. If $\gamma^{m_{0}}=\gamma_{1}^{m_{1}} \cdot \gamma_{2}^{m_{2}} \cdot \ldots \cdot \gamma_{n}^{m_{n}}, m_{0}, m_{1}, \ldots, m_{n} \in Z, m_{0} \neq 0$, define $\phi_{\gamma}: X \rightarrow \boldsymbol{R}$ by

$$
\phi_{y}(x)=\frac{m_{1}}{m_{0}} \phi_{\gamma_{1}}(x)+\frac{m_{2}}{m_{0}} \phi_{\gamma_{2}}(x)+\cdots+\frac{m_{n}}{m_{0}} \phi_{\gamma_{n}}(x), \quad x \in X .
$$

Then $\phi_{\gamma \gamma^{1}}=\phi_{\gamma}+\phi_{\gamma^{1}}, \gamma, \gamma^{1} \in \hat{Y}$. Also

$$
\begin{aligned}
\sum_{i=1}^{n} \phi_{\gamma_{i}}(T x) w_{i} & =\mathscr{R} U\left(f(T x) w_{j_{0}}\right)=\mathscr{R} U D_{M}\left(f(x) w_{j_{0}}\right) \\
& =M \sum_{i=1}^{n} \phi_{\gamma_{i}}(x) w_{i}=\sum_{i=1}^{n} \phi_{B \gamma_{i}}(x) w_{i} .
\end{aligned}
$$

Therefore $\phi_{B \gamma_{i}}(T x)=\phi_{B \gamma_{i}}(x), x \in X, 1 \leqq i \leqq n$, and hence $\phi_{\gamma}(T x)=\phi_{B \gamma}(x), x \in X, \gamma \in \hat{Y}$. By Theorem 2 there exists a continuous mapping $u: X \rightarrow Y$ such that $\gamma(u(x))$ $=\exp \left[i \phi_{\gamma}(x)\right], x \in X, \gamma \in \hat{Y}, u(T x)=B u(x), x \in X$, and $u$ is homotopic to a constant. Let $g: X \rightarrow Y$ be defined by $g(x)=c+C(x)+u(x), x \in X . g(T x)=c+C(T x)+u(T x)$ $=S(c+C(x))+B u(x)=S g(x), x \in X$.

It remains to show that $g$ maps $X$ onto $Y$. Suppose $X=\operatorname{inv} \lim \left(X_{q}, \tau_{q}\right)$ and $Y=\operatorname{inv} \lim \left(Y_{m}, \sigma_{m}\right)$ where the $X_{q}(q \geqq 1)$ are finite-dimensional tori and the $Y_{m}$ $(m \geqq 1)$ are $n$-dimensional tori. Suppose the given character $\delta \in \hat{X}_{k_{0}}$. Then each mapping $\phi_{y}: X \rightarrow \boldsymbol{R}$ only depends on $X_{k_{0}}$ and therefore $u$ only depends on $X_{k_{0}}$ and is homotopic to a constant by a homotopy depending only on $X_{k_{0}}$ (Theorem 2). The fact that $g$ maps $X$ onto $Y$ now follows from Lemma 2 .

We now show that if $h$ is given to be a homeomorphism then $g$ can be chosen to be a homeomorphism. Since we are assuming $h=c+C, C$ will be an isomorphism of $X$ onto $Y$. Let $g_{t}: X \rightarrow Y, t \in[0,1]$, be defined by $g_{t}(x)=c+C(x)+u_{t}(x), x \in X$, where $u_{t}: X \rightarrow Y$ satisfies $\gamma\left(u_{t}(x)\right)=\exp \left[i t \phi_{\gamma}(x)\right], x \in X, \gamma \in \hat{Y}$ (Theorem 2). By Lemma $2 g_{t}$ is a continuous mapping of $X$ onto $Y$ and $g_{t} T=S g_{t}, t \in[0,1]$. We shall show that $g_{t}$ is one-to-one for sufficiently small $t$.

It suffices to show that $g_{t} \circ C^{-1}: Y \rightarrow Y$ is one-to-one for sufficiently small $t$. We have $g_{t} \circ C^{-1}(y)=c+y+u_{t} \circ C^{-1} y, y \in Y$. Let $k$ be the smallest integer for which $\delta \circ C^{-1} \in \hat{Y}_{k}$, where $\delta$ is the given element of $\hat{X}$. By the definition of $\phi_{y}$, 
$\gamma \in \hat{Y}$, each $\phi_{\gamma} \circ C^{-1}$ can be considered as a real-valued function of $Y_{k}$, and therefore induces a mapping $P_{\gamma}: \boldsymbol{R}^{n} \rightarrow \boldsymbol{R}$ defined by $P_{\gamma}(v)=\phi_{\gamma} \circ C^{-1}\left(y_{v}\right), v \in \boldsymbol{R}^{n}$, where $y_{v}$ is any point of $Y$ which has component $v+Z^{n}$ in $Y_{k}$. Since each $P_{y}$ is a linear combination of sines and cosines of the coordinates of $\boldsymbol{R}^{n}$, there exists a constant $N$ such that if $\beta$ is a generator of any $\hat{Y}_{m}(m \geqq 1)$ then

$$
\left|P_{\beta}(v)-P_{\beta}\left(v^{\prime}\right)\right| \leqq N\left\|v-v^{\prime}\right\|, \quad v, v^{\prime} \in \boldsymbol{R}^{n},
$$

where $\|\cdot\|$ denotes the usual norm in $\boldsymbol{R}^{n}$.

Choose $t_{0} \in[0,1]$ so that $n t_{0} N<1$. Let $y, y^{\prime} \in Y, y \neq y^{\prime}$. Suppose $y=\left(y_{1}, y_{2}, \ldots\right)$, $y^{\prime}=\left(y_{1}^{\prime}, y_{2}^{\prime}, \ldots\right)$ where $y_{i}, y_{i}^{\prime} \in Y_{i}, i \geqq 1$. We shall show that $g_{t_{0}} \circ C^{-1}(y) \neq g_{t_{0}}$ $\circ C^{-1}\left(y^{\prime}\right)$. If $y_{k}=y_{k}^{\prime}$ then $\phi_{\gamma} \circ C^{-1}(y)=\phi_{\gamma} \circ C^{-1}\left(y^{\prime}\right), \gamma \in \hat{Y}$, and therefore $u_{t_{0}} \circ C^{-1}(y)$ $=u_{t_{0}} \circ C^{-1}\left(y^{\prime}\right)$. Hence $g_{t_{0}} \circ C^{-1}(y)-g_{t_{0}} \circ C^{-1}\left(y^{\prime}\right)=y-y^{\prime} \neq 0$. Now suppose $y_{k} \neq y_{k}^{\prime}$. Considering $Y_{k}$ as an $n$-torus let $\beta_{1}, \beta_{2}, \ldots, \beta_{n} \in \hat{Y}_{k}$ be defined by $\beta_{j}\left(z_{1}, \ldots, z_{n}\right)$ $=\exp \left(2 \pi i z_{j}\right)$. Define $G: Y_{k} \rightarrow Y_{k}$ by

$$
G\left(z_{1}, \ldots, z_{n}\right)=\left(z_{1}+t_{0} \phi_{\beta_{1}} \circ C^{-1}\left(y_{z}\right), \ldots, z_{n}+t_{0} \phi_{\beta_{n}} \circ C^{-1}\left(y_{z}\right)\right)+Z^{n}
$$

where $y_{z}$ is any point of $Y$ having $z=\left(z_{1}, \ldots, z_{n}\right)$ as its component in $Y_{k}$. By Lemma 3 , since $t_{0}$ is chosen so that $n t_{0} N<1$, we have that $G$ is one-to-one. Since $y_{k} \neq y_{k}^{\prime}$, $G\left(y_{k}\right) \neq G\left(y_{k}^{\prime}\right)$, i.e. $\beta_{j}\left(y_{k}+u_{t_{0}} \circ C^{-1}(y)\right) \neq \beta_{j}\left(y_{k}^{\prime}+u_{t_{0}} \circ C^{-1}\left(y^{\prime}\right)\right)$ for some $j$. Therefore $y+u_{t_{0}} \circ C^{-1}(y) \neq y^{\prime}+u_{t_{0}} \circ C^{-1}\left(y^{\prime}\right)$, i.e. $g_{t_{0}} \circ C^{-1}(y) \neq g_{t_{0}} \circ C^{-1}\left(y^{\prime}\right)$.

The following is a direct consequence of Theorems 3 and 6 .

COROllary 6.1. Let $X$ be a c.c.m.a. group and let $Y$ be a c.c.m.a. n-dimensional group. Let $T=a+A$ be an affine transformation of $X$ and $S=b+B$ an affine transformation of $Y$ for which there exists a continuous mapping $h$ of $X$ onto $Y$ satisfying $h T=S h$. There exists a nonaffine continuous mapping $g$ of $X$ onto $Y$ such that $g T=S g$ if and only if there exists $\delta \in \hat{X}, \delta \neq 1$, and a root $\lambda$, with $|\lambda|=1$, of the annihilating polynomial of $\hat{Y}$ with respect to $B$ such that $A^{p} \delta=\delta$ for some $p \geqq 1$ and $\delta(a+A(a)+$ $\left.\cdots+A^{p-1}(a)\right)=\lambda^{p}$ for all such $p$. If $h$ is a homeomorphism, the above conditions are necessary and sufficient for the existence of a nonaffine homeomorphism $g$ of $Y$ such that $g T=S g$.

If $B$ is an endomorphism of a c.c.m.a. $n$-dimensional group $Y$ onto $Y$ then it follows from the ergodicity conditions stated in $\S 1$ that $B$ is ergodic if and only if no root of the annihilating polynomial of $\hat{Y}$ with respect to $B$ is a root of unity. Moreover there is an element $\gamma \in \hat{Y}, \gamma \neq 1$ such that $B^{p} \gamma=\gamma$ if and only if the annihilating polynomial of $\hat{Y}$ with respect to $B$ has a $p$ th root of unity as a root.

TheOrem 7. Let $Y$ be a c.c.m.a. n-dimensional group and let $A$ and $B$ be endomorphisms of $Y$ onto $Y$. Suppose there exists a continuous mapping $h$ of $Y$ onto $Y$ such that $h A=B h$. There exists a nonaffine continuous mapping $g$ of $Y$ onto $Y$ such that $g A=B g$ if and only if $A$ and $B$ are not ergodic. If $h$ is given to be a homeomorphism then there exists a nonaffine homeomorphism $g$ of $Y$ such that $g A=B g$ if and only if $A$ and $B$ are not ergodic. 
Proof. If there exists a nonaffine continuous mapping $g$ of $Y$ into $Y$ satisfying $g A=B g$ then Theorem 3 asserts the existence of $\delta \in \hat{Y}, \delta \neq 1$, and a root $\lambda$ of the annihilating polynomial of $\hat{Y}$ with respect to $B$ such that $A^{p} \delta=\delta$ for some $p \geqq 1$ and $\lambda^{p}=1$ for all such $p$. Therefore $A$ and $B$ are not ergodic.

Conversely suppose $A$ and $B$ are not ergodic. Suppose $h$ is affine or there is nothing to prove. Let $h=c+C$, where $c \in Y$ and $C$ is an endomorphism of $Y$ onto $Y$. If $\hat{Y}$ is considered as an (additive) subgroup of $Q^{n}$ the nonsingular matrix representing $C$ is a conjugacy between the matrix representing $A$ and the matrix representing $B$. Hence the annihilating polynomial of $\hat{Y}$ with respect to $A$ is the same as the annihilating polynomial of $\hat{Y}$ with respect to $B$. Let $\delta \in \hat{Y}, \delta \neq 1$, be such that $A^{p} \delta=\delta$ for some $p \geqq 1$. Let $p$ be the least positive integer for which $A^{p} \delta=\delta$. Then the annihilating polynomial of $\hat{Y}$ with respect to $B$ has a root $\lambda$ which is a $p$ th root of unity. The result now follows from Theorem 6 .

We now give an example to show that Theorem 6 is false if the assumption that $\hat{Y}$ is finite-dimensional is replaced by the assumption that $\hat{Y}$ is polynomially annihilated by $B$, i.e. the converse of Theorem 3 is false.

Let $E$ denote the automorphism of the 4-torus $K^{4}$ determined by the matrix

$$
[E]=\left[\begin{array}{rrrr}
0 & 0 & 0 & -1 \\
1 & 0 & 0 & 8 \\
0 & 1 & 0 & -6 \\
0 & 0 & 1 & 8
\end{array}\right] .
$$

The matrix [ $E$ ] has two eigenvalues $\lambda_{1}, \bar{\lambda}_{1}$ of unit modulus which are not roots of unity and two distinct real eigenvalues $\lambda_{2}, \lambda_{3}$ [10]. Let $W$ denote the one-sided direct sum of an infinite number of copies of $\boldsymbol{K}^{4}$. Let $Y=\boldsymbol{K}+W . Y$ is an infinitedimensional torus. Let $S=b+B: Y \rightarrow Y$ be defined by

$$
S\left(y_{0}, y_{1}, y_{2}, \ldots\right)=\left(b_{0}, 0,0, \ldots\right)+\left(y_{0}, E y_{1}, y_{1}+E y_{2}, y_{2}+E y_{3}, \ldots\right)
$$

$y_{0} \in K, y_{i} \in K^{4}(i \geqq 1)$, where $\exp \left[2 \pi i b_{0}\right]=\lambda_{1}$. It is not difficult to show that $S$ is ergodic and $\hat{Y}$ is polynomially annihilated by $B$. The characteristic polynomial of $[E]$ is the minimal annihilating polynomial with respect to $B$ of some of the elements of $\hat{Y}$ and $\lambda_{1}$ is a root of this polynomial. If $\delta \in \hat{Y}$ is defined by

$$
\delta\left(y_{0}, y_{1}, y_{2}, \ldots\right)=\exp \left[2 \pi i y_{0}\right]
$$

then $B \delta=\delta$ and $\delta(b)=\lambda_{1}$. Hence (with $X=Y$ and $T=S$ ) all the assumptions of Theorem 6 (except that $Y$ be finite-dimensional) are satisfied by this example. However, we shall show that every continuous mapping commuting with $S$ is affine.

Suppose $g S=S g$ where $g$ is continuous. Let $g_{n}(n \geqq 0)$ be the projection of $g$ onto the $n$th factor in the representation $Y=K+K^{4}+K^{4}+\cdots, g_{0}$ is a continuous mapping of $Y$ into $K$ and $g_{n}(n \geqq 1)$ are continuous mappings of $Y$ into $K^{4}$. We shall show that each $g_{n}(n \geqq 0)$ is affine and this implies $g$ is affine. By Theorem 1 there 
exists a homomorphism $\mu_{0}: Y \rightarrow K$ and a continuous mapping $\phi_{0}: Y \rightarrow R$ such that $g_{0}(y)=\mu_{0}(y)+\phi_{0}(y)+Z$. Since $g_{0}(S y)=b_{0}+g_{0}(y)$ we have

$$
\phi_{0}(S y)=\phi_{0}(y)+a, \quad y \in Y, \quad \text { where } a \in \boldsymbol{R} .
$$

Therefore $\phi_{0}(y)-\int_{Y} \phi_{0}(y) d m$ ( $m$ denotes Haar measure on $Y$ ) is an invariant function under $S$ and therefore constant. Hence $\phi_{0}$ is constant and $g_{0}$ is affine. Suppose that some $g_{n}(n \geqq 1)$ is nonaffine. Let $k$ be the least integer for which $g_{k t}$ is nonaffine. By Theorem 1 there exist homomorphisms $\mu_{i}: Y \rightarrow K$ and continuous mappings $\phi_{i}: Y \rightarrow \boldsymbol{R}(1 \leqq i \leqq 4)$ such that

$$
g_{k}(y)=\left[\begin{array}{l}
\mu_{1}(y)+\phi_{1}(y) \\
\mu_{2}(y)+\phi_{2}(y) \\
\mu_{3}(y)+\phi_{3}(y) \\
\mu_{4}(y)+\phi_{4}(y)
\end{array}\right]+Z^{4}
$$

Since $g S=S g$ we have $g_{k} S=g_{k-1}+E g_{k}$ and $g_{k+1} S=g_{k}+E g_{k+1}$. Since $g_{k-1}$ is affine the uniqueness in Theorem 1 gives

$$
\left[\begin{array}{l}
\phi_{1}(S y) \\
\phi_{2}(S y) \\
\phi_{3}(S y) \\
\phi_{4}(S y)
\end{array}\right]=E\left[\begin{array}{l}
\phi_{1}(y) \\
\phi_{2}(y) \\
\phi_{3}(y) \\
\phi_{4}(y)
\end{array}\right]+e, \quad y \in Y, \quad \text { where } e \in \boldsymbol{R}^{4} \text {. }
$$

Let $D$, with matrix

$$
[D]=\left[\begin{array}{llll}
\lambda_{1} & 0 & 0 & 0 \\
0 & \bar{\lambda}_{1} & 0 & 0 \\
0 & 0 & \lambda_{2} & 0 \\
0 & 0 & 0 & \lambda_{3}
\end{array}\right]
$$

be the Jordan normal form of $E$ and let $U: C^{4} \rightarrow C^{4}$ be the linear transformation such that $U^{-1} E U=D$. By the type of argument used in the proof of Theorem 3 it follows that

$$
\left(\begin{array}{l}
\phi_{1}(y) \\
\phi_{2}(y) \\
\phi_{3}(y) \\
\phi_{4}(y)
\end{array}\right)=U\left(\begin{array}{c}
c \delta(y) \\
d \delta^{-1}(y) \\
0 \\
0
\end{array}\right)+e^{\prime}, \quad y \in Y
$$

where $c, d \in C$ and $e^{\prime} \in \boldsymbol{R}^{4}$. By Theorem 1 again

$$
g_{k+1}(y)=\left(\begin{array}{l}
\mu_{5}(y)+\phi_{5}(y) \\
\mu_{6}(y)+\phi_{6}(y) \\
\mu_{7}(y)+\phi_{7}(y) \\
\mu_{8}(y)+\phi_{8}(y)
\end{array}\right), \quad y \in Y
$$


where $\mu_{i}: Y \rightarrow \boldsymbol{K}$ are homomorphisms and $\phi_{i}: Y \rightarrow \boldsymbol{R}$ are continuous $(5 \leqq i \leqq 8)$. Since $g_{k+1}(S y)=g_{k}(y)+E g_{k+1}(y)$ we have

$$
\left(\begin{array}{l}
\phi_{5}(S y) \\
\phi_{6}(S y) \\
\phi_{7}(S y) \\
\phi_{8}(S y)
\end{array}\right)=E\left(\begin{array}{l}
\phi_{5}(y) \\
\phi_{6}(y) \\
\phi_{7}(y) \\
\phi_{8}(y)
\end{array}\right)+U\left(\begin{array}{c}
c \delta(y) \\
d \delta^{-1}(y) \\
0 \\
0
\end{array}\right)+e^{\prime \prime}, \quad y \in Y,
$$

where $e^{\prime \prime} \in \boldsymbol{R}^{4}$. Apply $U^{-1}$ to this equation and set

$$
\left(\begin{array}{l}
f_{1}(y) \\
f_{2}(y) \\
f_{3}(y) \\
f_{4}(y)
\end{array}\right)=U^{-1}\left(\begin{array}{l}
\phi_{5}(y) \\
\phi_{6}(y) \\
\phi_{7}(y) \\
\phi_{8}(y)
\end{array}\right)
$$

Then $f_{1}(S y)=\lambda_{1} f_{1}(y)+c \delta(y)+c^{1}$, where $c^{\prime} \in C$. Since $f_{1} \in L^{2}(Y)$ let $f_{1}(y)$ $=\sum_{i} a_{i} \gamma_{i}(y)\left(L^{2}\right.$ convergence) where $\gamma_{i} \in \hat{Y}$ and $\sum_{i}\left|a_{i}\right|^{2}<\infty$. If $\delta=\gamma_{i_{0}}$ then $a_{i_{0}} \delta(b)$ $=\lambda_{1} a_{i_{0}}+c$, and since $\delta(b)=\lambda_{1}$ this gives $c=0$. Consideration of the equation for $f_{2}$ implies $d=0$. Therefore $\phi_{i}, 1 \leqq i \leqq 4$, are constant and $g_{k}$ is affine, a contradiction. Therefore each $g_{n}(n \geqq 0)$ is affine.

Thus we have shown that every continuous mapping commuting with $S$ is affine.

We shall now state, without proof, a generalization of Theorem 6 . If $B$ is an endomorphism of a c.c.m.a: group $Y$ onto $Y$ we denote by $\hat{Y}(B, \lambda)$ the subgroup of $\hat{Y}$ generated by those elements of $\hat{Y}$ whose minimal annihilating polynomials with respect to $B$ have $\lambda$ as a root.

THEOREM 8. Suppose $X$ and $Y$ are c.c.m.a. groups, $T=a+A$ an affine transformation of $X$, and $S=b+B$ an affine transformation of $Y$ such that $\hat{Y}$ is polynomially annihilated by $B$. Suppose there exists a continuous mapping $h$ of $X$ onto $Y$ such that $h T=S h$. Also assume there exists $\delta \in \hat{X}, \delta \neq 1$, and a complex number $\lambda$ with $|\lambda|=1$, such that $\hat{Y}(B, \lambda)$ is a subgroup of $\hat{Y}$ of finite rank with the properties that $A^{p} \delta=\delta$ for some $p \geqq 1$ and $\delta\left(a+A(a)+\cdots+A^{p-1}(a)\right)=\lambda^{p}$ for all such $p$. Then there exists a nonaffine continuous mapping $g$ of $X$ onto $Y$ such that $g T=S g$. If $h$ is a homeomorphism then $g$ can be chosen to be a homeomorphism.

\section{REFERENCES}

1. L. M. Abramov, Metric automorphisms with quasi-discrete spectrum, Izv. Akad. Nauk SSSR 26 (1962), 513-530= Amer. Math. Soc. Transl. (2) 39 (1964), 37-56.

2. D. Z. Arov, On topological conjugacy of automorphisms and translations of compact abelian groups, Uspehi Mat. Nauk 18 (1963), no. 5 (113), 133-138.

3. F. J. Hahn, On affine transformations of compact abelian groups, Amer. J. Math. 85 (1963), 428-446. 
4. F. J. Hahn and W. Parry, Minimal dynamical systems with quasi-discrete spectrum, J. London Math. Soc. 40 (1965), 309-323.

5. P. R. Halmos, Lectures on ergodic theory, Chelsea, New York, 1956.

6. A. H. M. Hoare and W. Parry, Semi-groups of affine transformations, Quart. J. Math. Oxford Ser. 17 (1966), 106-111.

7. _- Affine transformations with quasi-discrete spectrum, J. London Math. Soc. 41 (1966), 88-96.

8. K. Hoffman and R. Kunze, Linear algebra, Prentice-Hall, Englewood Cliffs, N. J., 1961.

9. E. R. VanKampen, On almost periodic functions of constant absolute value, J. London Math. Soc. 12 (1937), 3-6.

10. P. Walters, Topological conjugacy of affine transformations of tori, Trans. Amer. Math. Soc. 131 (1968), 40-50.

The University of California,

Berkeley, California 